\title{
CROTALARIA VESPERTILIO (PAPILIONOIDEAE, LEGUMINOSAE) NUEVO REGISTRO PARA EL NORDESTE DE ARGENTINA Y ESTE DE PARAguay
}

\author{
HERNÁN G. BACH ${ }^{1,2,3}$ y RENÉE H. FORTUNATO1, 4, 5
}

\begin{abstract}
Resumen: Crotalaria vespertilio (Papilionoideae, Leguminosae) es citada, descripta e ilustrada por primera vez para NE de Argentina y E de Paraguay. Esta especie es afín a C. paulina, diferenciándose por caracteres florales. Para su reconocimiento se presenta una clave morfológica y además se incluye sinonimia con designación de lectotipos y un mapa de distribución geográfica actualizada para ambas especies en Sudamérica.
\end{abstract}

Palabras claves: Leguminosae, Crotalaria, taxonomy, South America.

Summary: Crotalaria vespertilio (Papilionoideae, Leguminosae) a new record from northeastern Argentina and eastern, described and illustrated. This species is similar to C. paulina, but differs by its floral morphology. A key separating it is included. In addition, synonyms with lectotypes here designed and an actualized distribution map in South America from both species are provided.

Key words: Leguminosae, Crotalaria, taxonomía, Sudamérica

\section{INTRODUCCIÓN}

El género pantropical y subpantropical Crotalaria L. está representado por más de 700 especies (Le Roux et al., 2013), encontrándose ca. de 90 taxones en el continente americano distribuidos desde el $\mathrm{S}$ de Estados Unidos de América hasta el $\mathrm{N}$ y centro de Argentina y W y N de Uruguay (Bernal, 1986; Windler, 1974; Windler \& Mclaughlin, 1980; Izaguirre \& Beyhaut, 1998; Gómez Sosa, 2000; Flores et al., 2006). En el Sur de Sudamérica se han registrado 22 especies (Fortunato et al., 2008), de las cuales 14 fueron citadas para Argentina agrupadas por el número de folíolos por

1 Instituto de Recursos Biológicos-CIRN-INTA. De Los Reseros y Nicolás Repetto s/n. Hurlingham, Pcia. Bs. As. E-mail: fortunato.renee@inta.gob.ar;

2 Cátedra de Botánica Agrícola FAUBA;

3 Cátedra de Farmacobotánica FFyB-UBA;

4 Concejo Nacional de Investigaciones Científicas y Técnicas (CONICET);

${ }^{5}$ Facultad de Agronomía y Ciencias Agroalimentarias, Universidad de Morón. hoja, 5 con hojas 3-folioladas: C. chaco-serranensis H. Bach \& Fortunato, C. incana L., C.maypurensis Kunth, C. micans Link, C. pumila Ortega y 9 con hojas 1 -folioladas: $C$. balansae Micheli, $C$. martiana Benth. subsp. mohlenbrockii (Windler \& S. G. Skinner) Planchuelo, C. paulina Schrank, $C$. pilosa Mill., C. sagittalis L., C. spectabilis Roth, $C$. stipularia Desv., $C$. vespertilio Benth. y $C$. tweediana Benth. (Gómez Sosa, 2000; Flores \& Miotto, 2005; Flores \& Tozzi, 2008; Bach \& Fortunato, 2010).

Durante la revisión del género para el Sur del continente, en el material con hojas 1-folioladas proveniente de las provincias de Misiones y Corrientes en el NE de Argentina, se encontraron especímenes que no concordaban en la morfología floral con la descripción de C. paulina. Esta especie ha sido citada para Argentina y Paraguay por Gómez Sosa (2000). A través de la consulta de las diagnosis y material tipo de los taxones relacionados se ha podido determinar que corresponde a $C$. vespertilio. Como antecedente se indica que Flores \& Tozzi (2008), si bien han señalado s u presencia en Argentina basándose en lo previamente citado, en el estudio realizado de los patrones fitogeográficos del 
género Crotalaria en Brasil, sólo la indican en tablas de ambiente, hábitat y distribución, sin citar material de referencia, precisar su ubicación geográfica, ni señalar los caracteres que posibilitan distinguirla en la región (Flores \& Tozzi, 2008: 480481). Sobre esta base en la presente contribución se describe e ilustra a $C$. vespertilio y su afín $C$. paulina, se indican los caracteres que permiten diferenciarlas, se verifica la sinonimia indicando la lectotipificación de los binomios y se señala la distribución geográfica actualizada.

\section{MATERIALES Y MÉTOdOS}

Se estudiaron los ejemplares depositados en los herbarios BAB, BAF, CTES, K, LP, MBM, MO, P, NY, SI (http://sweetgum.nybg.org/ih/). El estudio morfológico se llevó a cabo sobre material herborizado con microscopio estereoscópico (Willd M5 64482); y se consultaron las descripciones originales y ejemplares tipo y fototipos disponibles. Los mapas de distribución fueron digitalizados de las muestras de herbario, de la bibliografía citada y de las imágenes provistas en las páginas web: Field Museum of Natural History (http://emuweb.fieldmus eum.org/botany/ResultsList.php), Conservatoire et Jardin Botaniques de la Ville de Genève (http://www .ville-ge.ch/musinfo/bd/cjb/chg/search.php?lang=en), Kew Royal Botanic Gardens (http://apps.kew.org/her bcat/navigator.do), Missouri Botanical Garden (http:/ /www.tropicos.org/), Muséum National d'Histoire Na turelle (http://coldb.mnhn.fr/ScientificName/), New York Botanical Garden(http://sciweb.nybg.org/scien ce2/vii2.asp), y Naturhistorisches Museum Wien (http://herbarium.univie.ac.at/database/search.php), previa verificación de determinación del espécimen.

\section{RESULTADOS}

Polhill (1968, 1982) y Bisby \& Polhill (1973) agruparon a $C$. vespertilio y $C$. paulina en la sección Calycinae Wight \& Arn. por presentar flores con el extremo de la carina revoluto. Le Roux et al. (2013) basados en caracteres morfológicos y moleculares proponen una nueva clasificación infragenérica del género Crotalaria modificando en parte los caracteres diagnósticos de la sección
Calycinae: vexilo con callosidades lameliformes, carina redondeada en el tercio inferior, retorcida en el ápice, ápice levemente lobado, generalmente bilabiado, cáliz del largo de la carina a más largo y hojas simples, raramente trifolioladas. De acuerdo a la propuesta de Le Roux et al. (2013) las dos especies aquí estudiadas deben ser incluidas en la sección Calycinae Wight \& Arn. emend. M. M. le Roux \& B.-E. van Wyk.

Clave para diferenciar a Crotalaria vespertilio de $C$. paulina

1. Brácteas y bractéolas no foliáceas de $0,5-0,8 \mathrm{~cm}$ de largo, triangulares; bractéolas insertas en el ápice del pedicelo.

\section{C. vespertilio}

1'. Brácteas y bractéolas foliáceas de 1,5-2,5 cm de largo, lanceoladas; bractéolas insertas en la base del cáliz.

\section{C. paulina}

\section{Crotalaria vespertilio Benth.}

Annals of Natural History 3: 429. 1839. Typus: Brasil Goiás. Ad. Ourofino \& S. Cruz in C. Goyaz. - 1836 fl., J B E Pohl 1634 (holotypus K! K000500545; isotypus F! V0077385F; NY! NY6697; W! W0031489, visto en imagen digital) (Fig. 1, 3).

Arbustos o subarbustos de 1-2 m de alt. Tallos cilíndricos, glabros, a v eces alados por estípulas dilatadas a lo largo de los entrenudos. Estípulas ausentes o persistentes, heteromorfas, decurrentes de 2-10 cm long. x 1-5 cm lat., de ápice redondeado o falcado, pubescentes en el margen. Hojas 1folioladas, pecíolos de 4-7 $\mathrm{mm}$ long., vellosos; folíolos de 8-12 cm long. x 2,5-4 cm lat., elípticos a lanceolado-obovados, mucronados, cara adaxial glabra y abaxial séricea. Inflorescencias terminales, multifloras de 20-60 de long.; flores de 3-3,3 cm long.; brácteas de 5-8 mm long. x 1 -2 mm lat., persistentes, triangulares, pubescentes; pedicelos de 1-1,5 cm long., glabros; bractéolas de 5-6 mm long x $1 \mathrm{~mm}$ lat., persistentes, insertas en el receptáculo o 
H. G. Bach y R. H. Fortunato - Primer Registro Crotalaria vespertilio en Argentina y Paraguay

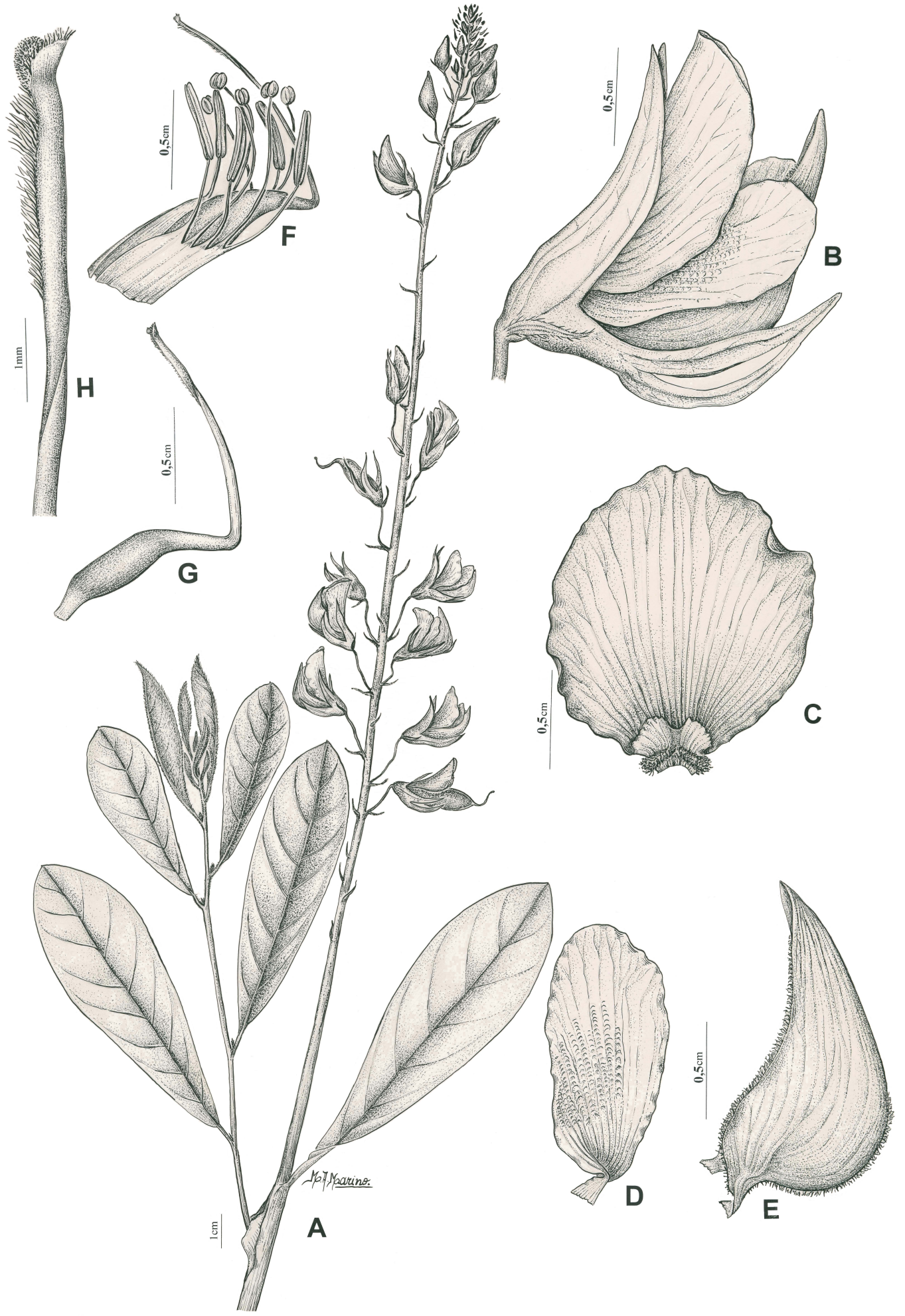

Fig. 1. Crotalaria vespertilio. A: Aspecto general de la planta. B: Flor. C: Estandarte. D: Ala. E: Carina. F: Androceo. G: Gineceo. H: detalle de estilo y estigma. De M. Morales 659 (BAB), dibujado por A. Marino. 
ápice del pedicelo, triangulares, pubescentes en el margen. Cáliz 5-lobado, glabro, 2-2,5 cm long., tubo de 4-5 mm long., lóbulos de $\pm 10 \mathrm{~mm}$ long., subiguales, los vexilares libres, el inferior y uno de los laterales concrescentes al ápice. Corola con vexilo de $2,5 \mathrm{~cm}$ long. x $2 \mathrm{c} \mathrm{m}$ lat., obovado a subcircular, apéndices alados de contorno irregular, barbado en el tercio inferior, levemente unguiculado, con márgenes pubescentes; alas de 1,6-2 cm long. $\mathrm{x}$ 0,6-1 cm lat., espatuladas a subrectangulares; carina de 1,9-2 cm long. x 0,7-1 cm lat., pubescente a lo largo del margen superior y en la porción basal del margen inferior, ápice convoluto. Gineceo con ovario cortamente estipitado, glabro; estilo geniculado, piloso debajo del estigma. Legumbre de 3-4 cm long. x 0,8-1 cm lat., glabras cortamente estipitadas. Semillas de 3-4 mm long. x $3 \mathrm{~mm}$ lat., reniformes, castaño-oscuras.

Distribución geográfica: Se encuentra desde el centro y S de Brasil, E de Paraguay hasta el NE de Argentina.

Hábitat: Crece en pastizales y márgenes de ríos desde los 250 a $780 \mathrm{msm}$.

Fenología: Florece de noviembre a marzo y fructifica de enero hasta abril/mayo.

Obs. 1: En el protólogo de C. vespertilio, Bentham citó Pohl $\mathrm{s} / \mathrm{n}^{\circ}$ basándose en el ejemplar estudiado (K), no obstante, en los isotipos depositados en F, NY y W indican como número: Pohl 1634. Es de señalar que la colección original de Pohl se encuentra en Naturhistorisches Museum Wien y sobre esa base en esta contribución de actualiza la numeración de los ejemplares tipo (holotipo e isotipos).

Obs. 2: Bentham (1859) ha citado que C. vespertilio se puede confundir con $C$. paulina cuando no presenta estipulas ovadas, persistentes; asimismo, la afinidad morfológica entre ambas especies ha sido menciona por Flores \& Miotto $(2001$; 2005) y Avendaño (2011).

Obs.3: Si bien en la base http://www.tropicos.org/ Name/13029615?tab=specimens se $r$ egistra la presencia de esta especie en América Central: Guatemala y Honduras, es de indicar que los especímenes de referencia corresponden a u na determinación errónea, y por lo tanto se restringe su distribución al continente Sudamericano.
Obs. 4: En la Argentina y Paraguay C. vespertilio crece preferentemente en ambientes perturbados (ej.: borde de caminos), coincidiendo con lo citado por Flores \& Miotto (2005) quienes asociaron a la actividad antrópica la discontinuidad e ncontrada entre las poblaciones de los Estados de Brasil (Mato Grosso, Santa Catarina y Paraná).

Material examinado. BRASIL. Edo. Goiás: São João d'Aliança. Serra Geral do Paraná, ca. $3 \mathrm{~km}$ S.of São João de Aliança, near Riacho, 14 43' 38" S, 47 31' 59" W, 15-III-1971, H. Irwin et al. 31845 (NY). Distrito Federal: Brasília, Fercal, $15^{\circ} 46^{\prime} 47^{\prime \prime}$ S, $47^{\circ} 55^{\prime} 46^{\prime \prime} \mathrm{W}, 20-\mathrm{V}-1967$, E. Heringer 11517 (NY). Edo. Mato Grosso: Xavantina-Cachimbo road, $65 \mathrm{Km}$. from Xavantina. Cerrado, 25-V-1966, D. R. Hunt \& J. Ferreira Ramos 5570 (P); C/ 5 Km. of Xavantina $52^{\circ} 20^{\prime} \mathrm{W}, 14^{\circ} 44^{\prime} \mathrm{S}, 28-\mathrm{VII}-1967$, J. A. Ratter \& J. Ramos R.211 (P); Mun. Rondonopolis, Serra da Patrolina, 13-II-1974, G. Hatschbach 34148 (P). PARAGUAY. Dpto. Alto Paraná, Pto. Bertoni, Costa del río Paraná 54 $35^{\circ}$ W, $25^{\circ}$ 40’ S, 31-III-1993, A. Schinini et al. 28284 (CTES). ARGENTINA. Prov. Misiones: Dpto. Candelaria, Puerto Santa Ana, 17-II-2008, M. Morales et al. 659 (BAB); Dpto. Iguazú, Libertad puerto Bemberg, bajada al Río Paraná 14-VII-1992, R. A. Rossow et al. 4810 (BAF, BAB); Parque Nacional Iguazú, Isla San Martín 25 $41^{\prime}$ S, 54 46' W, 4-III-1995, F. O. Zuloaga et al. 5258 (CTES, SI); Dpto. Posadas, Playa del Río Paraná, I-1919, Spegazzini 612 (LP 23479); Dpto. San Ignacio, Santo Pipó margen del Río Paraná, 5-II-2011, R. D. Almada et al. 113 (CTES, MNES). Prov. Corrientes: Dpto. Ituzaingó, Fiplasto Empresa Forestal, centro Forestal Villa Olivari borde de carrizal, frente al cuadro 130. 12-XII-1994, S. Tressens et al. 5155 (CTES, BAB).

\section{Crotalaria paulina Schrank}

Pl. Rar. Hort. Monac.: 88. 1822. Typus: Brasil: Patria Brasilia, ubi D. de Martius ad Urbem S. Pauli semina Legit. P1. 88. 1822. (Lectotypus, designado aquí: P1. 88. 1822. Fotocopia BAB!) (Fig. 2, 3).

Crotalaria racemosa Vell. Fl. Flumin. 306. 1825 (= 1829). Typus: Habitat fruticetis mediterraneis. (Lectotypus, designado aquí: Icon.7, táb. 106. 1825 (=1829). 


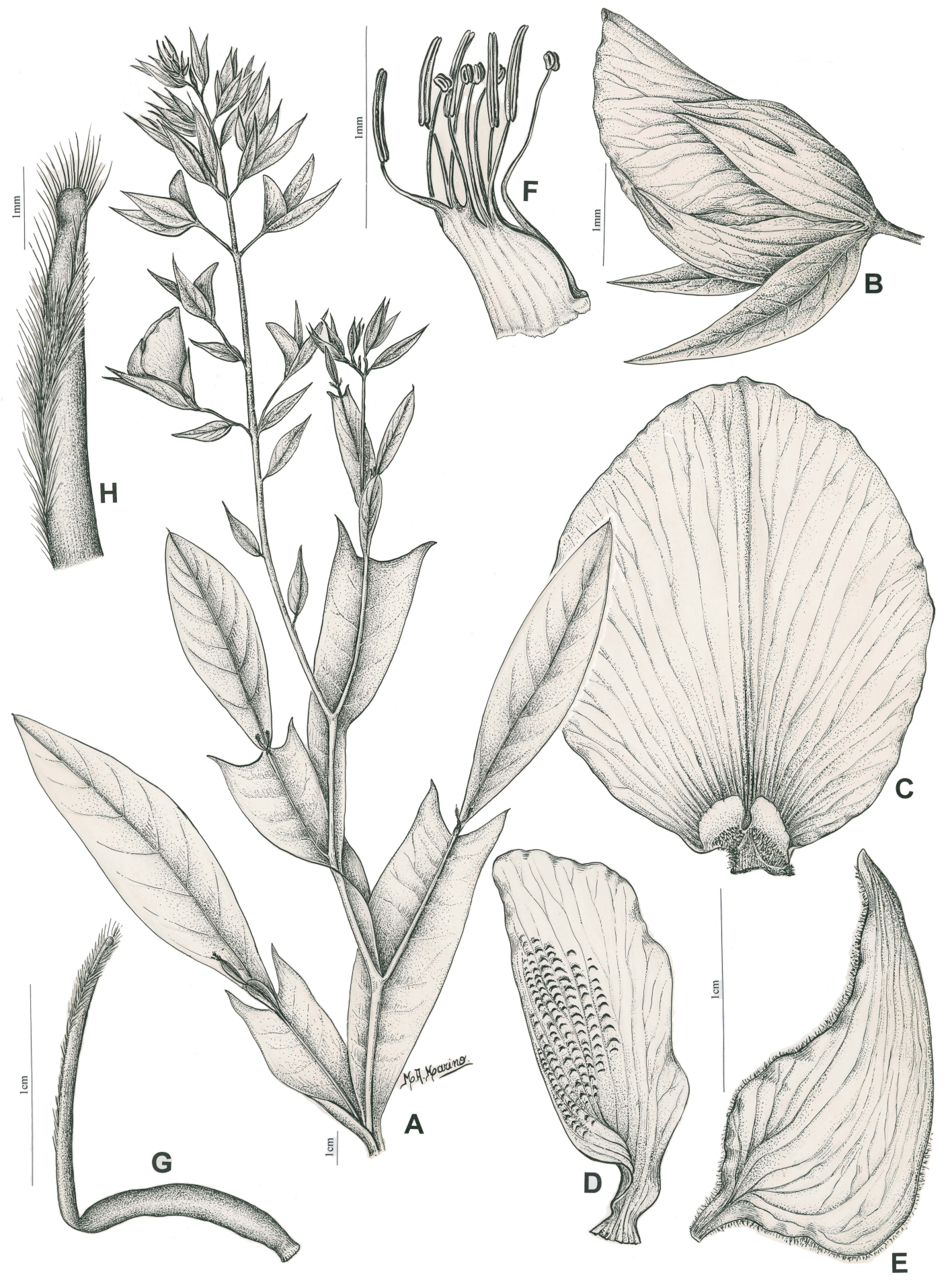

Fig. 2. Crotalaria paulina. A: Aspecto general de la planta. B: Flor. C: Estandarte. D: Ala. E: Carina. F: Androceo. G: Gineceo. H: detalle de estilo y estigma. De R. Fonnegra et al. 2434 (LP), dibujado por A. Marino 


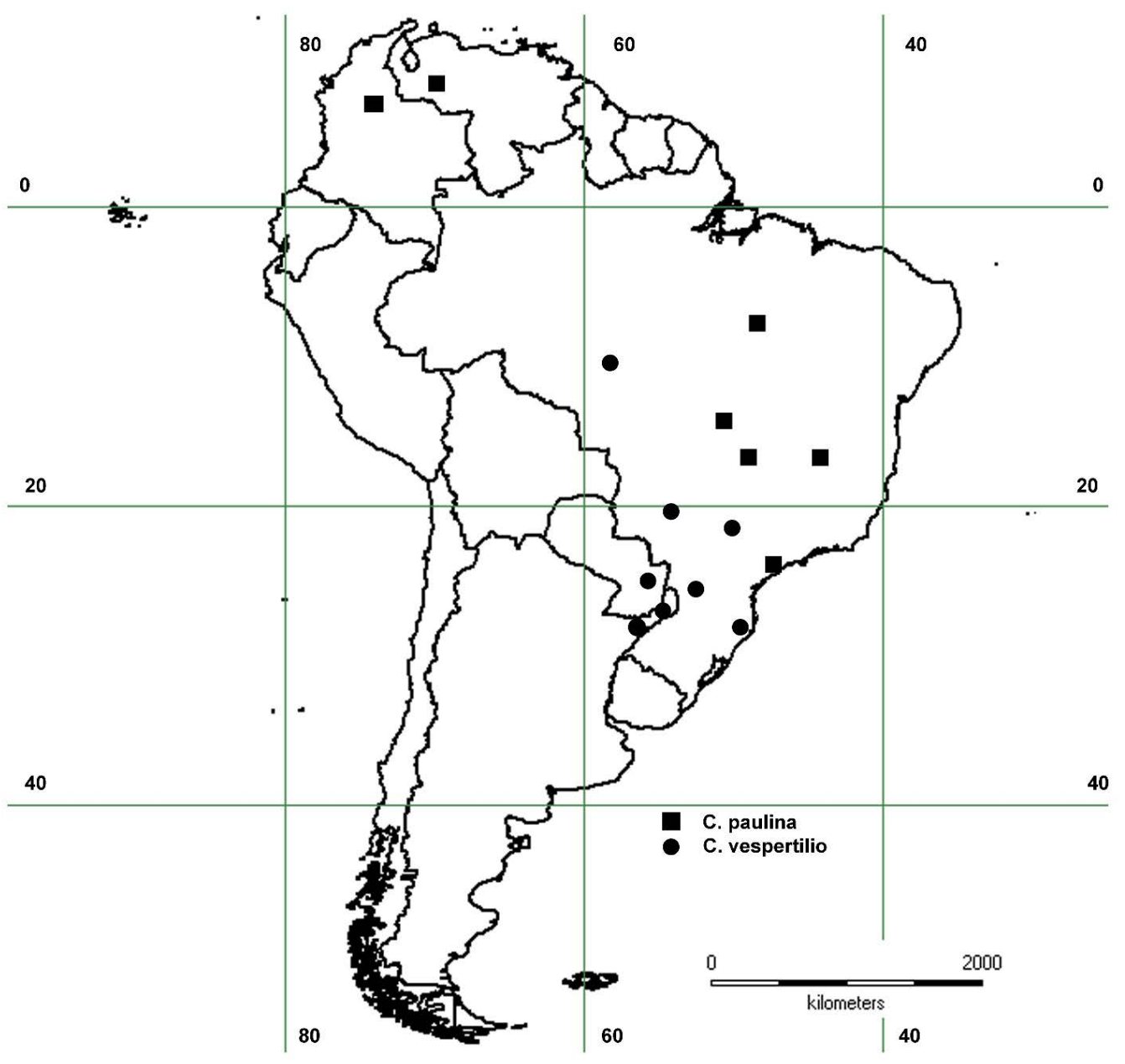

Fig. 3. Mapa con la distribución de C. paulina y C. vespertilio.

Crotalaria bracteosa Benth. Ann. Nat. Hist. 3: 428. 1839. Typus: Brasil, J. Lhotsky 91 (Lectotypus, designado aquí: W! W-Endlicher 0031494, visto imagen digital).

Crotalaria virgata Benth. Ann. Nat. Hist. 3: 429. 1839. Typus: Brasil: Minas Gerais, Eugenho Abrantes Franc. Oliveira, Pohl s/n $\mathrm{n}^{\mathrm{o}}$; Langsdorff $\mathrm{s} / \mathrm{n}^{\mathrm{o}}$ (Lectotypus, designado aquí: Pohl 2693, W! WHerb.bras. 0031493, Isolectotypus: G! G00364972; K! K000500560, visto imagen digital).
Arbustos de hasta $3 \mathrm{~m}$ alt. Tallos cilíndricos, glabros. Estípulas ausentes o persistentes, dimorficas, decurrentes de ápice redondeado o falcado; hojas unifolioladas foliolos de 9-15 cm long. $x$ 3-5 cm lat., elípticos a 1 anceolados, obovados, cara adaxial glabra, y abaxial pubescente. Inflorescencias de $50 \mathrm{~cm}$ o más de long.; flores de 2$6 \mathrm{~cm}$ long., brácteas de 1-2,5 cm long. x 0,5-0,8 cm lat., persistentes, lanceoladas, foliaceas; pedicelos de 1-2cm long., glabros; bractéolas 1-2,5 cm long. $\mathrm{x}$ $0,5-0,8 \mathrm{~cm}$ lat., persistentes, insertas en la base del 


\section{H. G. Bach y R. H. Fortunato - Primer Registro Crotalaria vespertilio en Argentina y Paraguay}

cáliz, lanceoladas, foliáceas. Cáliz 5-lobado, glabro, 2-2,5 cm long., tubo 0,4-0,5 cm long., lóbulos 1-2$\mathrm{cm}$ long., formando dos grupos (2 superiores y 3 inferiores) concrescentes en el ápice. Corola con vexilo eliptico-obovado, apéndices alados de contorno regular, barbado debajo de los mismos; alas espatuladas a subrectangulares; carina 1-2 cm. de long., pubescente a lo largo del margen superior, ápice convoluto. Ovario cortamente estipitado, glabro; estilo geniculado, piloso debajo del estigma. Legumbre 4-6 cm long. x 0,8-1 cm lat., glabras cortamente estipitadas. Semillas $5 \mathrm{~mm}$ long. x $3 \mathrm{~mm}$ lat., reniformes, castañas.

Distribución geográfica: En Sudamérica presenta distribución disyunta en el NW de Venezuela, W de Colombia y Centro y SE de Brasil.

Hábitat: Praderas y borde de bosque en galerías entre los 500 y 1800 msm (Bernal, 1986; Avendaño, 2011).

Fenología. Florece y fructifica de noviembre a mayo.

Obs. 1: De acuerdo al Código Internacional de Nomenclatura de Algas, Hongos y Plantas: Arts. 9.2. y 9.9. se designan como lectotipos a las ilustraciones de C. paulina y C. racemosa.

Obs. 2: En el protólogo de C. bracteosa, Bentham (1839) cita el ejemplar Lhotsky sin número de la colección. De los herbarios consultados en donde se ha informado están depositados los especímenes recolectados por Lhotsky en Brasil (Stafleud \& Cowan, 1981), solo se ha encontrado en el herbario W el ejemplar: Lhotsky 91 que concuerda con la descripción original además de poseer la determinación específica con letra de Bentham: WEndlicher 0031494. Hasta el presente el material original visto por Bentham no ha sido encontrado.

Obs. 3: La diagnosis original de C. virgata fueron citados los sintipos: Pohl $\mathrm{s} / \mathrm{n}^{\mathrm{o}}$ y Langsdorff $\mathrm{s} / \mathrm{n}^{\mathrm{o}}$ (Bentham, 1839). A través del estudio del binomio solamente ha sido posible registrar la colección de Pohl en los herbarios de $\mathrm{G}, \mathrm{K}$ y W, designando al ejemplar depositado en $\mathrm{W}$ como lectotipo por ser el más completo, representado por ramas con flores. Si bien en el protólogo del binomio no se menciona el número de colección, en $\mathrm{W}$ es donde están depositados los especímenes originales de Pohl y el material asignado como lectotipo posee los datos de la localidad citada en la descripción y corresponde a Pohl 2693.

Obs. 4: Si bien C paulina no crece en la región se incluye la descripción, sinonimia, ilustración y mapa de distribución dado que es frecuentemente confundida con su afín $C$. vespertilio y hasta el presente ha sido citada en Argentina por Gómez Sosa (2000), Flores \& Miotto (2005) y Flora del Conosur Catalogo de Plantas Vasculares http://www2.darwin.edu.ar/Proyectos/FloraArgentin a/DetalleEspecie.asp? forma $=\&$ variedad $=\&$ subespeci $\mathrm{e}=\&$ especie $=$ paulina $\&$ genero $=$ Crotalaria\&espcod $=3$ 0886.

Material examinado. COLOMBIA. Dpto. Antioquia: Jardín-Andes (10,7 Km before Andes) $05^{\circ} 37^{\prime} \mathrm{N}, 75^{\circ} 51^{\prime} \mathrm{W}, 31-\mathrm{II}-1988$, J. L. Zarucchi et al. 7022 ( SI, MO); Municipio Andes, Vereda La Pava, $14 \mathrm{Km}$., Carretera Andes hacia La Vereda El Líbano, 23-VIII-1988, R. Fonnegra et al. 2434 (HUA, LP). Dpto. Tolima: Chaparral, Granja Camilo Iriarte, 16-IV-1987, L. M. Quiñones 1083 (MO). VENEZUELA. Edo. Mérida: Dry slope, sandy, 24-II-1964, F. J. Breteler 3622 (P). Edo. Portuguesa: margen derecho del río Guanare, 31VIII-1985, A. Licata 16 (PORT). BRASIL. Edo. Piaui: -1841, G. Gardner 2525 (K). Edo. Goiás: -III1840/1841, G. Gardner 3648 (K); Chapada dos Veadeiros, $12 \mathrm{~km}$ south of Cavalcante, 9-III-1969, Irwin et al. 24165 (B, NY); Corumbá de Goiás. Serra dos Pirineus, ca. $20 \mathrm{~km} \mathrm{E}$. of Pirenópolis, $15^{\circ} 51^{\prime} 00^{\prime \prime} \mathrm{S}, 48^{\circ} 45^{\prime} 48^{\prime \prime} \mathrm{W}, 16-\mathrm{I}-1972, \mathrm{H}$. S. Irwin et al. 34299 ( NY). Edo. Minas Gerais: Bello Horizonte, Estação de Barreiro, $7 \mathrm{Km}$ south of Belo Horizonte, 26-V-1945, L. O. Williams \& V. Assis 7128 (SI); Distrito Carangola, Trail Areponga to Fazenda de Grama, about 2 1/2 legues Capoera, 26-I1930, Y. Mexia 4230 (P). Distrito Federal: In the area of the Córrego Cabeça de Veado 9,5 Km. SSE of Brasília TV Tower, 15-VI- 1976, J. A. Ratter et al. R. 3170 (P); Rio Torto, immediately N. of Brasília, D.F., 18-IX-1965, H. S. Irwin et al. 8443 (MO); Bacia do Rio São Bartolomeu, $15^{\circ} 35^{\prime} 26$ " S, $47^{\circ} 35^{\prime}$ 29" W, 22-V-1980, E. P. Heringer 4893 (MO); Brasília, Parque do Guará, $15^{\circ} 46^{\prime} 47^{\prime \prime} \mathrm{S}$, $47^{\circ} 55^{\prime} 46^{\prime \prime}$ W, 1-V-1974, E. P. Heringer 13280 (NY, SP). Edo. São Paulo: Grounds of the Instituto Botanico, 23 38' 29" S, 46 37' 38" W, 21-II-1976, G. Davidse 10469 (MO); Parque do Estado e Jardim Botânico de São Paulo, a beira da picada da estrada 
de acesso ao prédio novo. $23^{\circ} 32^{\prime} 25^{\prime \prime} \mathrm{S}$, 46 37' 48"W, 28-III-1964, W. Hoehne 5966 (NY, $\mathrm{SP})$.

\section{AgRADECIMIENTOS}

Se agradece a los curadores de los herbarios aquí citados, así como a la ilustradora científica del Instituto de Recursos Biológicos, Sra. A. Marino por la realización de las ilustraciones.

\section{BIBLIOGRAFÍA}

AVENDAÑO, N. 2011. Revisión taxonómica del género Crotalaria L. (Faboideae-Crotalarieae) en Venezuela. Acta Bot. Venez. 34 (1): 13-27.

BACH, H. G. \& R. H. F ORTUNATO. 2010. Crotalaria chaco-serranensis H. Bach \& Fortunato nom. Nov. (Leguminosae) del Chaco argentino. Candollea 65: 291-300.

BENTHAM, G. 1859. Crotalaria. In: Martius, C.F.P., A. C. Endlicher \& J. Urban (eds.), Fl. Bras. 15 (1): 17-32.

BISBY, F. A. \& R. M. POLHILL. 1973. The role of taximetrics in angiosperm taxonomy II. Parallel taximetric and orthodox studies in Crotalaria L. New Phytol. 72: 727-742.

BERNAL, M. H. 1986. Crotalaria. In: Pinto, P. \& P. Ruiz (eds.), Flora de Colombia 4: 1-118. Bogotá.

BURKART, A. 1952. Crotalaria. Las Leguminosas Argentinas Silvestres y Cultivadas. Ed. 2, pp. 326-329. Ediciones Acme, Buenos Aires.

FLORES, A. S. \& S. T. S. MIOTTO. 2001. O gênero Crotalaria L. (Leguminosae-Faboideae) na Região Sur do Brasil. Iheringia Bot. 55: 189-247.

FLORES, A. S. \& S. T. S. MIOTTO. 2005. Aspectos Fitogeográficos das espécies de Crotalaria L. (Leguminosae-Faboideae) na região sul do Brasil. Acta Botânica Brasília 19: 245-249.

FLORES, A. S. \& A. M. G. A. TOZZI. 2008. Phytogeographical patterns of Crotalaria species (Leguminosae-Papilionoideae) in Brazil. Rodriguesia 59: 477-486.

FORTUNATO, R. H., S. T. S. MIOTTO, P. IZAGUIRRE \& R. BEYHAUT. 2008. Fabaceae. In: Zuloaga, F. O., O. Morrone \& M. J. Belgrano (eds.), Catálogo de las Plantas Vasculares del Cono Sur. Monogr. Syst. Bot. Missouri Bot. Gard. 107: 2166-2170.

GÓMEZ-SOSA, E. 2000. Las especies argentinas de Crotalaria (Leguminosae - Crotalarieae): Novedades, Descripciones y Clave. Gayana Bot. 57: 67-87.

IZAGUIRRE, P. \& R. BEYHAUT. 1999. Crotalaria. Las Leguminosas en Uruguay y regiones vecinas. Papilionoideae 1: 454-463. Editorial Agropecuaria Hemisferio Sur, Montevideo.

LE ROUX, M. M., J. S. BOATWRIGHT \& B. E. VAN WYK. 2013. A global infrageneric classification system for the genus Crotalaria (Leguminosae) based on molecular and morphological evidence. Taxon 62: 957-971.

POLHILL, R. M. 1968. Miscellaneous notes on African species of Crotalaria. II. Kew Bull. 22: 169-348.

POLHILL, R. M. 1982. Crotalaria in Africa and Madagascar: 389 pp. A. A. Balkema, Rotterdam.

STAFLEU, F. A. \& R. S. COWAN, 1981. Taxonomic literature. A selective guide to botanical publications and collections with dates, commentaries and types III. Bohn, Scheltema and Holkema, Utrecht.

WINDLER, D. R. 1974. A systematic treatment of the native unifoliolate Crotalaria of North America (Leguminosae). Rhodora 76: 151204.

WINDLER, D. R. \& L. MCLAUGHLIN. 1980. Crotalaria. In: Woodson Jr., R. E. \& R. W. Schery (ed.), Flora of Panamá. Ann. Missouri Bot. Gard. 67: 599-613.

Recibido el 15 de octubre de 2013, aceptado el 2 de Mayo de 2014. 